\title{
Immunodominance: a new hypothesis to explain parasite escape and host/parasite equilibrium leading to the chronic phase of Chagas' disease?
}

\author{
M.M. Rodrigues, B.C.G. de Alencar, C. Claser and F. Tzelepis \\ Centro Interdisciplinar de Terapia Gênica, Escola Paulista de Medicina, Universidade Federal de São \\ Paulo, São Paulo, SP, Brasil
}

Correspondence to: M.M. Rodrigues, CINTERGEN, EPM, UNIFESP, Rua Mirassol, 207, 04044-010 São Paulo, SP, Brasil

\begin{abstract}
Intense immune responses are observed during human or experimental infection with the digenetic protozoan parasite Trypanosoma cruzi. The reasons why such immune responses are unable to completely eliminate the parasites are unknown. The survival of the parasite leads to a parasite-host equilibrium found during the chronic phase of chagasic infection in most individuals. Parasite persistence is recognized as the most likely cause of the chagasic chronic pathologies. Therefore, a key question in Chagas' disease is to understand how this equilibrium is established and maintained for a long period. Understanding the basis for this equilibrium may lead to new approaches to interventions that could help millions of individuals at risk for infection or who are already infected with $T$. cruzi. Here, we propose that the phenomenon of immunodominance may be significant in terms of regulating the host-parasite equilibrium observed in Chagas' disease. $T$. cruzi infection restricts the repertoire of specific T cells generating, in some cases, an intense immunodominant phenotype and in others causing a dramatic interference in the response to distinct epitopes. This immune response is sufficiently strong to maintain the host alive during the acute phase carrying them to the chronic phase where transmission usually occurs. At the same time, immunodominance interferes with the development of a higher and broader immune response that could be able to completely eliminate the parasite. Based on this, we discuss how we can interfere with or take advantage of immunodominance in order to provide an immunotherapeutic alternative for chagasic individuals.
\end{abstract}

Key words: Chagas' disease; Trypanosoma cruzi; Immunodominance; Major histocompatibility complex

Research supported by FAPESP (\#2006/1983-4), The Millennium Institute for Vaccine Development and Technology (CNPq, \#420067/2005-1) and The Millennium Institute for Gene Therapy. F. Tzelepis (\#2004/110106-6), B.C.G. de Alencar (\#2003/ 09675-9) and C. Claser (\#2003/09672-0) are recipients of Doctorate fellowships from FAPESP. M.M. Rodrigues is the recipient of a fellowship from CNPq (\#307151/2006-9).

Received June 3, 2008. Accepted October 29, 2008

Chagas' disease is an acute or chronic illness caused by the digenetic protozoan parasite Trypanosoma cruzi. Currently, there are more than 15 million chronically infected individuals in the Americas. These individuals will carry the parasite for life in a process in which parasite and host reach a life-long equilibrium. This type of equilibrium greatly benefits the parasite because it increases the time for successful transmission to the blood feeding Triatominae host. In a group of individuals ( $30 \%)$, this equilibrium will be broken and the individuals will develop the symptomatic forms of the disease characterized by damage of the heart and/or autonomic denervation of the digestive tract.

Parasite-host equilibrium leads to parasite persistence, which is considered to be the most likely cause of the chagasic chronic pathologies $(1,2)$. Therefore, a key question in Chagas' disease is to understand how this equilibrium is established, allowing both to survive for a long period. Understanding the basis for this equilibrium may lead to new interventions that could potentially help millions of individuals at risk for infection and chronically 
infected with $T$. cruzi. We propose that the phenomenon of immunodominance may be quite significant in terms of regulating the host-parasite equilibrium observed in Chagas' disease. In addition, we discuss how we can interfere with or take advantage of immunodominance in order to provide an immunotherapeutic alternative for individuals at risk for infection or already infected with $T$. cruzi.

Following initial infection with $T$. cruzi, the vast majority of individuals survive acute infection due to the presence of adaptive immune responses mediated by class I and class II major histocompatibility complex (MHC-I and MHC-II) restricted $\mathrm{T}$ cells. Studies in experimental models have been instrumental in establishing that the removal of MHCI or MHC-II restricted T cells leads to extreme susceptibility and mouse death following acute infection (3). In spite of the protective immune responses, sterile immunity is rarely achieved in chagasic individuals and parasite survival within the host establishes a life-long chronic infection.

Adaptive host immune responses are dependent on MHC molecules. Pathogens have a number of potential amino acid sequences that can bind to MHC-I or -II molecules and provide targets for specific T cells. On the other hand, hosts have a vast number of T cell receptors (TCR) that are clonally distributed on these $T$ cells to recognize a variety of MHC-peptide complexes. In spite of this large number of possible combinations, pathogen-specific $\mathrm{T}$ cells seem to preferentially recognize a small number of epitopes. This preference, known as immunodominance, may be due to different mechanisms including the formation of stable MHC-peptide complexes on the surface of

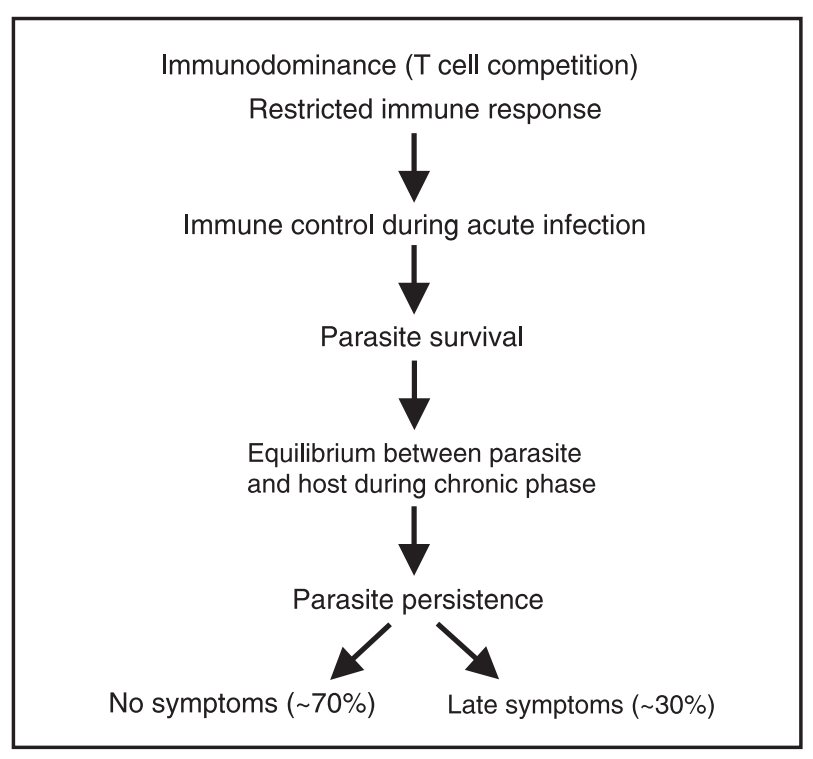

Figure 1. New theory to explain the chronic phase of Chagas' disease. antigen presenting cells, higher amounts or a higher affinity of specific $T$ cell precursors and competition of T cells for antigen presenting cells (4-6).

In the case of self-resolving common acute viral or bacterial infections, immunodominance focuses the response on a few determinants. It has been proposed (6) that this strategy successfully maximizes the power of effector immune responses and, at the same time, minimizes the risk of auto-immunity. The phenomenon of immunodominance most likely evolves from the interaction between vertebrate immune systems and these common acute viral and bacterial diseases. However, in the case of chronic infections such as Chagas' disease, the impact of immunodominance is completely obscure and may even be harmful to the host.

It was initially considered to be difficult to conduct studies on the immunodominance of $\mathrm{T}$ cell immune responses during $T$. cruzi infection due to a large parasite genome, which contains more than 12,000 genes. Nevertheless, two recent studies have described immunodominant epitopes recognized by murine and human CD8 T cells $(7,8)$. During the acute phase of Chagas' disease, immunodominance may have an impact similar to that observed in the viruses and bacteria causing acute infections, as it guarantees host survival. However, on the negative side, immunodominance may not allow for an immune response that is broad enough to completely eliminate the parasites. In that case, it would be a key factor restricting the magnitude of the immune response, allowing parasite survival and providing the means to establish the parasite/host equilibrium observed during the chronic life-long infection (see Figure 1).

The fact that immunodominance restricts specific immune responses to $T$. cruzi poses the question of who could benefit from such a restriction. A limitation on the magnitude and specificity of the immune response could be important for the host to avoid excessive immunopathology. We have evidence against this hypothesis. Following vaccination with plasmid DNA or recombinant proteins, we generated memory CD8 T cells that displayed a very fast anamnestic immune response after a challenge with $T$. cruzi (7). These cells were specific for immunodominant CD8 epitopes. In fact, it led to a reduced parasite development and prevented the establishment of chronic phase tissue pathologies. Also, some of these vaccinated mice completely cleared the parasite. Such a strong immune response did not cause any discernible immunopathology (9-11). However, the fact that the immune response of the vaccinated mice may be even more bias to the immunodominant epitope precludes conclusions on the immunopathological role of $\mathrm{T}$ cells of other specificity. 
Alternatively, immunodominance may hamper the development of stronger immune responses and aid the parasites in causing disease and establishing a chronic infection. This hypothesis is also supported by our vaccination studies in which we dramatically increase the immune response to these immunodominant epitopes following challenge. Under these circumstances, we obtained reduced parasitism and limited disease development. Also, in some cases, we reached sterile protection against $T$. cruzi, showing that vaccination could be a useful approach to boost the host immunodominant response (912). We concluded that aberrantly high immunodominant responses may, in some cases, clear the parasite from the host. Whether it occurs in non-immune naive individuals remains to be determined.

If immunodominance were indeed so critical for T. cruzi survival, a very powerful mechanism would have to be used to restrict the activation of $T$ cells specific to the nonimmunodominant epitopes. One of the most powerful mechanisms to establish immunodominance is the interference or competition between $\mathrm{T}$ cells restricted by distinct $\mathrm{MHC}$ molecules (cross-competition). This mechanism has been described in a number of experimental models; however, whether it really occurs during infections is still a matter of debate $(4-6,13)$.

While studying the mechanism underlying immunodominance during $T$. cruzi infection in mice, we found that a strong cross-competition occurs during the priming of $T$. cruzi-specific CD8 T cells. This mechanism is extremely powerful and severely narrows the immune response and generates immunodominance. Therefore, $T$ cells specific for a single epitope can block the development of immunity to several other epitopes, reducing the magnitude and breadth of the immune response and eventually favoring parasite escape (14).

This hypothesis, however, could create a severe bottleneck. The parasite requires at least one immunodominant epitope to be recognized by CD4 and CD8 T cells in each individual. In view of the variation of $\mathrm{MHC}$ and the number of mammals that $T$. cruzi infects, this could be a severe problem. For example, the lack of a CD8 T cell epitope could lead to a high parasitemia and to the death of the host in the acute phase before transmission. How can the presence of at least one epitope for every host MHC be ensured? The genome sequence of $T$. cruzi identified families of proteins that have up to 1340 genes. We propose that the gene duplication process helps to provide at least one immunodominant epitope for each mammalian host. Our proposal is supported by the observation that the immunodominant CD8 epitopes described so far are members of a larger family of genes, the trans-sialidase. Dis- tinct members of this family are recognized by CD8 T cells restricted by different mouse haplotypes $(7,8,12)$.

Many pathogens establish chronic infections as is the case for $T$. cruzi. Whether similar mechanisms contribute to the chronic process may be an important question to address. Some of these pathogens have a high mutation rate and, in these cases, the immune response relying mainly on few immunodominant CD8 ${ }^{+} \mathrm{T}$ cell epitopes may favor the escape of immune response associated with pathogen mutation or any particular antigenic variation process. An example supporting this hypothesis was described in a human viral infection with hepatitis $C$ virus. A strong immunodominant human CD8 $\mathrm{T}$ cell response was developed, causing an escape mutant to be selected (15). Whether dominant immune responses select for $T$. cruzi mutants has never been pursued in depth and should be evaluated in different experimental models and chagasic patients. However, a single recent report was unable to detect the presence of mutant parasites following vaccination of BALB/c mice with a $T$. cruzi trans-sialidase recombinant antigen (16).

Previous studies have suggested the presence of immunosuppression and/or polyclonal activation of the immune response during experimental $T$. cruzi infection $(17,18)$. Using concomitant immunization with recombinant adenovirus and infection with $T$. cruzi, we were unable to detect any immunosuppressive activity on the in vivo cytotoxic activity of $T$. cruzi-specific or non-specific CD8 ${ }^{+} \mathrm{T}$ cells (Mello $\mathrm{S}$, Rodrigues MM, unpublished results). Also, experiments in vivo failed to detect any polyclonal activation of $\mathrm{CD} 8^{+} \mathrm{T}$ cells. TCR transgenic CD8 ${ }^{+}$OT- 1 cells specific for ovalbumin were adoptively transferred to naive mice. Upon infection with $T$. cruzi, these cells failed to proliferate. This evidence strongly argues against in vivo polyclonal activation (19). The previously described polyclonal activation described most likely reflected activation of a large number of specific $T$ cells as proposed by Martin et al. (8).

We propose that immunodominance may be the strongest mechanism of escape used by $T$. cruzi. Based on that assumption, the means to break the deadlock is through increasing and/or broadening the immune responses prior to and/or during infection (9-11). Whether immunotherapy should focus on dominant, subdominant, or cryptic epitopes or on all of them at the same time can be addressed experimentally in the near future and may provide the basis for rational interventions.

\section{Acknowledgments}

The authors are in debt with Dr. V. Nussenzweig (NYU), Dr. Milena Soares (FIOCRUZ), and Dr. Karina R. Bastos (USP) for enthusiastic support and review of the manuscript. 


\section{References}

1. Tarleton RL. Chagas disease: a role for autoimmunity? Trends Parasitol 2003; 19: 447-451.

2. Kierszenbaum F. Where do we stand on the autoimmunity hypothesis of Chagas disease? Trends Parasitol 2005; 21: 513-516.

3. Rodrigues MM, Boscardin SB, Vasconcelos JR, Hiyane MI, Salay G, Soares IS. Importance of CD8 T cell-mediated immune response during intracellular parasitic infections and its implications for the development of effective vaccines. An Acad Bras Cienc 2003; 75: 443-468.

4. Sette A, Sundaran R. The phenomenon of Immunodominance: speculations on the nature of immunodominance. In: Frelinger JA (Editor), Immunodominance - the choice of the immune system. Weinheim: Wiley-VCH Verlag GmbH \& Co., KGaA; 2006. p 57-71.

5. Gaddis DE, Fuller MJ, Zajac AJ. CD8 T-cell immunodominance, repertoire, and memory. In: Frelinger JA (Editor), Immunodominance - the choice of the immune system. Weinheim: Wiley-VCH Verlag GmbH \& Co., KGaA; 2006. p 109-145.

6. Yewdell JW, Del Val M. Immunodominance in TCD8+ responses to viruses: cell biology, cellular immunology, and mathematical models. Immunity 2004; 21: 149-153.

7. Tzelepis F, de Alencar BC, Penido ML, Gazzinelli RT, Persechini PM, Rodrigues MM. Distinct kinetics of effector CD8+ cytotoxic $T$ cells after infection with Trypanosoma cruzi in naive or vaccinated mice. Infect Immun 2006; 74: 2477-2481.

8. Martin DL, Weatherly DB, Laucella SA, Cabinian MA, Crim MT, Sullivan S, et al. CD8+ T-Cell responses to Trypanosoma cruzi are highly focused on strain-variant trans-sialidase epitopes. PLoS Pathog 2006; 2: e77.

9. Vasconcelos JR, Hiyane MI, Marinho CR, Claser C, Machado AM, Gazzinelli RT, et al. Protective immunity against Trypanosoma cruzi infection in a highly susceptible mouse strain after vaccination with genes encoding the amastigote surface protein-2 and trans-sialidase. Hum Gene Ther 2004; 15: 878-886.

10. Araujo AF, de Alencar BC, Vasconcelos JR, Hiyane MI, Marinho CR, Penido ML, et al. CD8+-T-cell-dependent control of Trypanosoma cruzi infection in a highly susceptible mouse strain after immunization with recombinant proteins based on amastigote surface protein 2. Infect Immun 2005;
73: 6017-6025.

11. Machado AV, Cardoso JE, Claser C, Rodrigues MM, Gazzinelli RT, Bruna-Romero O. Long-term protective immunity induced against Trypanosoma cruzi infection after vaccination with recombinant adenoviruses encoding amastigote surface protein-2 and trans-sialidase. Hum Gene Ther 2006; 17: 898-908.

12. de Alencar BC, Araujo AF, Penido ML, Gazzinelli RT, Rodrigues MM. Cross-priming of long lived protective CD8+ T cells against Trypanosoma cruzi infection: importance of a TLR9 agonist and CD4+ T cells. Vaccine 2007; 25: 60186027.

13. Kastenmuller W, Gasteiger G, Gronau JH, Baier R, Ljapoci $\mathrm{R}$, Busch $\mathrm{DH}$, et al. Cross-competition of CD8+ $\mathrm{T}$ cells shapes the immunodominance hierarchy during boost vaccination. J Exp Med 2007; 204: 2187-2198.

14. Tzelepis F, de Alencar BC, Penido ML, Claser C, Machado $\mathrm{AV}$, Bruna-Romero $\mathrm{O}$, et al. Infection with Trypanosoma cruzi restricts the repertoire of parasite-specific CD8+ T cells leading to immunodominance. J Immunol 2008; 180: 1737-1748

15. Tester I, Smyk-Pearson S, Wang P, Wertheimer A, Yao E, Lewinsohn DM, et al. Immune evasion versus recovery after acute hepatitis $\mathrm{C}$ virus infection from a shared source. J Exp Med 2005; 201: 1725-1731.

16. Hoft DF, Eickhoff CS, Giddings OK, Vasconcelos JR, Rodrigues MM. Trans-sialidase recombinant protein mixed with CpG motif-containing oligodeoxynucleotide induces protective mucosal and systemic Trypanosoma cruzi immunity involving CD8+ CTL and B cell-mediated cross-priming. $J$ Immunol 2007; 179: 6889-6900.

17. Minoprio PM, Coutinho A, Joskowicz M, Imperio Lima MR, Eisen H. Polyclonal lymphocyte responses to murine Trypanosoma cruzi infection. II. Cytotoxic T lymphocytes. Scand J Immunol 1986; 24: 669-679.

18. Minoprio P, Itohara S, Heusser C, Tonegawa S, Coutinho A. Immunobiology of murine $T$. cruzi infection: the predominance of parasite-nonspecific responses and the activation of TCRI T cells. Immunol Rev 1989; 112: 183-207.

19. Martin D, Tarleton R. Generation, specificity, and function of CD8+ T cells in Trypanosoma cruzi infection. Immunol Rev 2004; 201: 304-317. 